\title{
Restoring brain function after stroke — bridging the gap between animals and humans
}

\author{
Nick S Ward ${ }^{1,2,3}$
}

${ }^{1}$ Sobell Department of Motor Neuroscience, UCL Institute of Neurology, 33 Queen Square, London WC1N 3BG.

${ }^{2}$ The National Hospital for Neurology and Neurosurgery, Queen Square, London WC1N 3BG.

${ }^{3}$ UCLPartners Centre for Neurorehabilitation, UCL Institute of Neurology, Queen Square, London WC1N 3BG

n.ward@ucl.ac.uk

\section{Biography}

Nick Ward is an academic neurologist at UCL Institute of Neurology and the National Hospital for Neurology and Neurosurgery, Queen Square, London, UK, where he works in the stroke and neurorehabilitation service. His research uses structural and functional brain imaging to study how reorganisation of brain networks supports recovery of upper limb movement after stroke. His goal is to understand the mechanisms of recovery so that we might predict both optimal treatments of upper limb impairment and long-term outcomes after stroke. 


\section{Abstract}

Stroke is the leading cause of complex adult disability in the world. Recovery from stroke is often incomplete, which leaves many people dependent on others for their care. The improvement of longterm outcomes should, therefore, be a clinical and research priority. As a result of the advances in our understanding of the biological mechanisms involved in recovery and repair after stroke, therapeutic opportunities to promote recovery through manipulation of post-stroke plasticity have never been greater. This work has almost exclusively been carried out in preclinical animal models of stroke with little translation into human studies. The challenge ahead is to develop a mechanistic understanding of recovery from stroke in humans. Advances in neuroimaging techniques now enable us to reconcile behavioural accounts of recovery with molecular and cellular ones. Consequently, clinical trials can be designed in a stratified manner that takes into account when an intervention should be delivered and who is most liable to benefit. This approach is expected to lead to a substantial change in how restorative therapeutic strategies are delivered in patients after stroke.

\section{Key points}

- Stroke is the leading cause of complex adult disability in the world, but currently we do not provide enough of the right physical or behavioural interventions to drive recovery

- Clear lesion-induced changes occur in brain structure and function early after stroke, which result in an environment with unique heightened plasticity that can support restoration of function, termed spontaneous biological recovery

- Intense, high-dose behavioural training aimed at the reduction of impairment and the restoration of function should be (but currently is not) delivered in this critical time window

- The basis of spontaneous biological recovery in humans is unclear, which yields uncertainty over how and when to augment or prolong this process with novel therapies - further characterization is required to enable realistic phase III trials 
- Human neuroimaging techniques combined with modelling approaches can provide the appropriate biomarkers with which to map out a mechanistic approach to understand who and when to treat

- The use of structural imaging to quantify damage in a range of brain regions can help predict long-term outcomes and provide the basis for stratification in restorative trials 
Almost 17 million people worldwide experience a first-time stroke each year ${ }^{1}$, which is equivalent to one new stroke every 2 seconds. Stroke mortality is declining ${ }^{2}$ but in the UK over 1 million people live with the consequences of stroke, of whom over one-third are dependent on others for their care. The epidemiological shift of stroke disease burden towards long-term conditions means that these numbers will continue to rise ${ }^{3}$ Often, the decline in functional abilities that takes place in many patients ${ }^{4}$ goes unrecognized, and so, unsurprisingly, the overall economic burden of stroke is high (estimated at over UK£9 billion a year in the UK). The fact that stroke is both a chronic and a progressive condition should influence research priorities in this area, but funding for research into stroke, and stroke recovery in particular, lags far behind cancer, coronary heart disease and dementia ${ }^{5}$. Improvement of recovery and long-term outcomes is an urgent clinical and scientific goal, but success is slow to materialize.

How are the most dramatic clinical improvements expected to be achieved? Care in the hyperacute and acute period after stroke has improved dramatically over the past two decades, but our attention must now turn to treatments that actively promote recovery. One reason for optimism is that work in animal models points to a time-limited period of heightened plasticity after focal brain injury. However, achieving the best possible outcomes in patients after stroke requires two key challenges to be addressed. The first is how to take advantage of this critical period through the optimal timing, intensity, amount and even type of behavioural training that makes up neurorehabilitation. This question has been discussed elsewhere but, in brief, studies support the use of intense training that focuses on reducing impairment in the first few weeks and months post-stroke to take advantage of biological repair mechanisms ${ }^{6}$. The second challenge, and the focus of this Review, is how to augment the biological mechanisms of post-stroke plasticity to enhance or prolong the effects of behavioural training in patients after a stroke. The translational nature of this question is important, because although work in preclinical animal models has been pivotal in highlighting the biological basis of recovery, as yet virtually no benefit has been observed for humans. I will discuss the reasons why this lack of benefit might be and the prospects for developing a mechanistic understanding of post-stroke plasticity in humans. In particular, exciting prospects exist for the development of human biomarkers that provide an appropriate intermediate level of mechanistic description with which to 
bridge the current explanatory gap between what we know about recovery from pre-clinical studies and human studies.

\section{Recovery after stroke is proportional}

A starting point for determining the biological basis of recovery in patients after a stroke is to ask why some patients fail to recover. Stroke is one of the most common causes of physical disability worldwide and $\sim 80 \%$ of stroke survivors experience impairment of movement on one side of the body $^{7}$. Hand and arm impairment in particular is often persistent, disabling ${ }^{8}$ and a major contributor to reduced quality of life. In one study, only $38 \%$ of patients who presented with an initially paralysed upper limb regained some dexterity by 6 months $^{9}$, and by 4 years two-thirds of patients perceived that loss of arm function was still a major problem ${ }^{10}$. These studies and many others clearly demonstrate that recovery is variable and difficult to predict. Factors associated with poor outcomes include right hemisphere damage, somatosensory deficit, visual inattention, homonymous hemianopia and urinary incontinence ${ }^{9,11}$. However, the dominant factor for predicting long-term upper limb outcome is initial severity of motor impairment. Additional factors that have independent predictive power over and above their association with this initial severity have not been identified.

The ability of initial severity to predict upper limb recovery was first quantified as the proportional recovery rule $[G]^{12}$. When applied to real clinical data, two key findings exist (FIG. 1$)$ that provide challenges but also opportunities for the field. The first is that initial upper limb impairment predicts later upper limb outcome extremely accurately in patients presenting with mild to moderate impairment. This result is disconcerting to those involved in post-stroke neurorehabilitation because it implies that any variability in the dose of rehabilitation delivered in the first 3 months exerts no substantial effect on a patient's level of motor impairment. The second key result is that proportional recovery fails in about half of patients presenting with severe impairment. In other words, in patients presenting with the same high level of initial severity, about half recover proportionately and half fail to make any substantial recovery (FIG. 1). Importantly, this finding tells us that the causes of initial impairment are probably independent from the biological factors that are important for the subsequent recovery process. This interpretation provides an opportunity, because factors important 
for recovery might represent targets for novel therapeutics that aim to optimize the biological factors that maximize the effects of behavioural training.

The proportional recovery rule has been confirmed in the motor domain several times ${ }^{12-15}$ and suggests two clear clinical questions. Firstly, how can we help patients with stroke to regain more than $70 \%$ of lost function and secondly, how can we turn poor recoverers into proportional recoverers? The answers to these questions will dramatically change our approaches to promoting recovery after stroke. Evidence for proportional recovery has also been shown for non-motor domains such as languag $\mathrm{e}^{16}$ and neglect ${ }^{17}$, and so this striking clinical phenomenon provides a novel and important model for investigating both potentially modifiable biological factors that are necessary for maximising recovery of function after stroke in humans, as well as currently non-modifiable factors that will help to make accurate predictions of long-term outcome.

\section{Spontaneous biological recovery}

Why do some patients experience poor recovery after stroke and yet others who are clinically indistinguishable have good recovery? The differences in these two groups manifest in the first few days and weeks after stroke. During this time there might (or might not) be a rapid generalized improvement in impairment that is in contrast to the modest gains that are made in the chronic phase ${ }^{18}$. Decades of work in animal models clearly show that a window of opportunity exists after focal brain damage within which behavioural training will have a much greater effect than outside the window. This early post-stroke phase has been described as a period of spontaneous biological recovery [G]. Early evidence of this critical period for recovery-related training was provided by Biernaskie and colleagues ${ }^{19}$ who found that rats that commenced motor training of the affected forelimb starting at 30 days post-stroke exhibited little improvement when compared with those whose treatment commenced earlier at 5-14 days post-stroke The causal role of the lesion itself in initiating spontaneous biological recovery was illustrated further by Zeiler and colleagues ${ }^{20}$ who showed that intensive reach training of a mouse commenced 7 days after stroke was not able to promote full recovery. However, when the same animal was given a second stroke and training was commenced 2 days later (presumably within the critical period), then recovery was substantially 
enhanced, and resulted in performance levels that approached those seen before either stroke. Clearly, focal brain damage sets in motion a series of biological events that, when combined with appropriate type and intensity of behavioural training ${ }^{6}$, can support dramatic recovery.

\section{Structural plasticity after stroke}

A substantial amount of work has been undertaken in animal models to define the molecular and cellular processes that underlie the formation of new local and large-scale brain circuits that support recovery from stroke. These studies are well described elsewhere ${ }^{21-25}$. Briefly, the basic elements of neural repair that can be seen in animal models of stroke include axonal sprouting, dendritic branching, synaptogenesis, neurogenesis and gliogenesis, and all can be enhanced in the early post-stroke period. Regeneration seems to occur in brain regions connected to the damaged area, including peri-infarct, ipsilesional and contralesional brain and spinal cord networks. Not all sprouting is clinically beneficial, and only axonal sprouting that links functionally related brain areas is consistently associated with improved post-stroke outcomes ${ }^{26}$. Definitive evidence of these restorative processes in humans is scarce, but markers suggestive of neurogenesis ${ }^{27}$, gliogenesis ${ }^{28}$ and axonal sprouting ${ }^{27}$ have been found in human post-stroke perilesional brain tissue. Consequently, the occurrence of similar biological responses to brain injury in both animals and humans seems probable.

The precise temporal and spatial ordering of these post-stroke biological events is governed by alterations in gene expression. Researchers have often remarked that the biological environment of the post-stroke brain resemble that of the developing brain, and that 'recovery recapitulates ontogeny'23. However, a clear distinction between regenerative and developmental transcriptomes has been shown, which indicates a unique regenerative molecular program at work ${ }^{29}$. Furthermore, expression of the regenerative transcriptome is strongly influenced by age at stroke onset, with earlier induction of growth-inhibiting molecules and later expression of growth-promoting molecules exhibited by older animals than by younger animals ${ }^{30}$. 
Preclinical work has attempted to both promote neuronal regeneration and, most commonly, to block extracellular inhibitory signals that counteract regeneration, with some successes $(\mathrm{BOX} 1)^{24,31}$. Changes to the structure of brain networks will not independently restore function, and all of these studies stress the need for appropriate levels of behavioural training, something that is often omitted from preclinical studies in animal models. The potential to form new functionally relevant circuitry that can be shaped by behavioural training provides a compelling mechanistic framework for functional recovery after stroke. However, the timing of administration of growth-promoting compounds, both in relation to the initial stroke damage and to the behavioural training itself, will clearly have a major effect on the therapeutic capacity. Whether training is delivered at the same time as growth-promoting molecules or sequentially could influence the type of sprouting that occurs and, consequently, whether behaviour is helped or hindered ${ }^{32}$. In addition, the effect that post-stroke behaviour can have on regenerative processes themselves is important to understand. For example, early compensatory use of the contralesional forelimb impairs recovery of the affected limb ${ }^{33}$, possibly through aberrant synaptogenesis in the perilesional cortex ${ }^{34}$. Any behaviour, if overtrained, will take advantage of the increased post-stroke potential for experience-dependent plasticity, and so abnormal or compensatory patterns of behaviour can become learned. Once again, this finding highlights the need for an appropriate form of behavioural training that can take advantage of any spontaneous or therapeutically enhanced potential for plasticity.

As well as asking 'when' treatment should be administered, 'where' is probably an equally important question. Most of the compounds discussed have been administered via intravenous or intrathecal routes, but accurate spatial and temporal delivery might both be necessary to achieve the desired outcomes. Advances made in the last few years in tissue engineering ${ }^{35,36}$ and optogenetics ${ }^{37}$ provide potential methods for precisely delivering regenerative molecules to functionally relevant brain regions.

\section{Functional plasticity after stroke}

Identification of the trigger for post-stroke regenerative processes could provide further therapeutic opportunities. In addition to the structural changes described above, focal brain damage results in 
alterations in neuronal excitability ${ }^{38}$. Immediately after stroke, signalling by the excitatory neurotransmitter glutamate is excitotoxic and contributes to cell death, whereas signalling by the inhibitory neurotransmitter GABA can counteract this toxicity through cell hyperpolarization ${ }^{39}$. This period lasts about 3 days post-stroke in the mouse ${ }^{40}$ and for an uncertain time in humans, after which the beneficial and detrimental effects of GABA and glutamate signalling seem to reverse. Specifically, changes to the cortical excitatory-inhibitory balance have long been known to influence the potential for experience-dependent plasticity in cortex and can reopen critical periods of plasticity in the adult brain ${ }^{41}$. Reduced inhibitory tone can lead to facilitation of downstream changes in neuronal structure ${ }^{42}$ and one possibility is that the altered levels of neuronal activity that result from a change in excitability regulate neurogenesis and the activity of growth factors (such as brain derived neurotrophic factor; BDNF) through epigenetic mechanisms ${ }^{43}$. Reduced cortical inhibitory mechanisms can lead to expanded and less specific receptive fields ${ }^{44,45}$, enhanced long-term potentiation $^{46}$ and remapping of sensorimotor functions to surviving cortex ${ }^{47}$ in both hemispheres ${ }^{48}$, all of which is potentially useful when functional reorganisation of residual post-stroke brain structures is important for recovery of normal function. An altered balance between inhibitory GABAergic and excitatory glutamatergic signalling in surviving stroke regions and networks could, therefore, be a key event that sets other restorative mechanisms in motion.

In 2009, Murphy and Corbett ${ }^{21}$ proposed that after the acute stroke period, attenuation of neuronal activity in brain regions connected to the damaged region might be reversed by a homeostatic increase in neuronal excitability, a process that can last at least several weeks ${ }^{21}$. Levels of neuronal excitability are determined by the balance in activity between GABA and glutamate, both of which are known to be altered after stroke ${ }^{38}$. For example, enhanced glutamate signalling through AMPA receptors, the major excitatory signalling system in the adult brain, is associated with improved recovery in stroke models ${ }^{49}$. This effect is probably due to downstream induction of BDNF ${ }^{49}$, which once again links altered neuronal excitability with downstream changes in axonal structure ${ }^{50}$. Much work on GABAergic signalling after stroke has focussed on the reduction in phasic (that is, synaptic) inhibition in the first few weeks after injury ${ }^{51}$ to increase the likelihood of long-term potentiation ${ }^{46}$. Specifically, GABA $A_{A}$ receptors are dowregulated ${ }^{48,52}$, and the density of a number of inhibitory 
interneurons is reduced after focal brain damage $\mathrm{e}^{44,53}$. Both increased glutamatergic signalling and reduced phasic GABAergic signalling would be consistent with the idea of a homeostatic restitution of neuronal activity ${ }^{21}$. However, two studies have suggested that increased perilesional tonic inhibitory signalling via extrasynaptic $G_{A B A}$ receptors might be the dominant response to stroke ${ }^{40,54}$. When this tonic inhibition was reversed (using an a5 subunit that contained an extrasynaptic $\mathrm{GABA}_{\mathrm{A}}$-receptor inverse agonist) motor outcomes improved in both mouse ${ }^{40}$ and rat $^{51}$ models of stroke. Although the increase in extracellular GABA in response to cerebral ischaemia is transient, the increase in tonic inhibitory signalling can persist for more than 1 month $^{38}$ making this therapeutic window attractive compared with the window available for reperfusion strategies.

The interactions between excitatory pyramidal cells and numerous inhibitory interneurons in the cortex is clearly complex and becomes more complex after stroke ${ }^{55}$. In addition, prolonged ischaemia affects different cell types unequally ${ }^{56}$ and causes alterations in the distribution of receptor subtypes $^{57}$. The numbers of inhibitory interneurons (some of which inhibit other inhibitory interneurons) and pyramidal cells, as well as the ratios of receptor subtypes in the surviving cortex are not only unclear, but can differ between individuals. Nevertheless, the weight of evidence from animal studies to date suggests that spontaneous biological recovery is either augmented by a homeostatic restitution of cortical activity secondary to reduced phasic GABAergic inhibitory signalling, or blocked by excessive tonic GABAergic inhibitory signalling. Beyond the hyperacute period (up to 3 days post-stroke), what follows at a cellular level suggests that alterations in cortical inhibitory and excitatory mechanisms are important to determine the potential for plasticity and downstream structural changes that support recovery. Consequently, components of these inhibitory and excitatory mechanisms represent exciting and novel therapeutic targets for enhancing behavioural training after stroke.

As with mechanisms of structural plasticity, the mechanisms responsible for the alterations in cortical excitatory-inhibitory balance that underlie changes in post-stroke functional plasticity are amenable to pharmacological and non-pharmcological manipulation. The most popular non-pharmacological approach is the use of non-invasive brain stimulation which appears to be able to enhance the effects of behavioural training to a small degree ${ }^{58,59}$. In a mouse model, direct current stimulation to the brain 
appeared to augment synaptic plasticity through BDNF dependent mechanisms ${ }^{60}$. However, in human studies it is not clear how much or how accurately electrical current is delivered to target brain regions and consequently results are inconsistent and potential mechanisms poorly understood 61,62 .

As described, tonic inhibition can be reversed by antagonists or inverse agonists of the a5-subunitcontaining extrasynaptic $\mathrm{GABA}_{\mathrm{A}}$ receptor, and compounds for use in humans are currently available and are under investigation in phase I studies. Zolpidem is an interesting pharmacological agent that binds with high affinity to a1-containing $\mathrm{GABA}_{\mathrm{A}}$ receptors through which it mediates sedative and hypnotic effects. However, zolpidem can also influence tonic inhibition through a5-containing $\mathrm{GABA}_{\mathrm{A}}$ receptors in a dose-dependent manner, such that low levels of the drug augment tonic inhibition and high levels reduce it ${ }^{63}$. Zolpidem can improve recovery in a mouse model of stroke ${ }^{64}$, and has been reported to mediate interesting effects such as the temporary reversal of deficits in language, cognitive and motor function in single patient cases with stroke ${ }^{65,66}$. However, given the uncertainty over how zolpidem works, the mechanism of recovery in these individuals remains unclear.

The idea that pharmacological approaches can help promote recovery of function after stroke has been well described ${ }^{67}$. Modulation of a number of neurotransmitter systems has shown positive effects in animal models of stroke, usually correlating with their effect on long-term potentiation ${ }^{67}$. A key message from this early work is that close temporal coupling of the drug and the behavioural training is required for maximum therapeutic effect, which suggests that the therapeutic mechanisms are short lived and reversible, rather than being due to chronic effects. This point has not always translated into study design, but should be considered when interpreting the results of a pharmacotherapy study.

The current interest in selective serotonin reuptake inhibitors (SSRIs) comes from the fluoxetine for motor recovery after acute ischemic stroke (FLAME) study in which $20 \mathrm{mg}$ fluoxetine daily, started 5-10 days after ischaemic stroke and continued for 3 months, enhanced upper-limb motor recovery ${ }^{68}$. Many smaller studies of SSRIs have similar findings, but heterogeneity between studies is high ${ }^{69}$. Although SSRIs can influence structural plasticity, compelling evidence supports a plasticity-modifying effect mediated through the GABAergic system. Chronic doses of fluoxetine can 
reinstate critical-period plasticity in adult rats through a reduction of extracellular levels of GABA and an increase in BDNF expression ${ }^{70}$. Furthermore, in a mouse model of stroke, $\mathrm{Ng}$ and colleagues $^{71}$ showed that fluoxetine treatment was able to prolong (but not reinstate) the critical period of poststroke plasticity through the reduction of inhibitory interneuron expression in intact cortex ${ }^{71}$. Serotonin can have inhibitory (via $5 \mathrm{HT}_{1 \mathrm{~A}}$ receptors) or facilitatory (via $5 \mathrm{HT}_{2 \mathrm{~A}}$ receptors) effects on pyramidal cells, but most fast-spiking inhibitory interneurons are inhibited by serotonin through $5 \mathrm{HT}_{1 \mathrm{~A}}$ receptors ${ }^{72}$. However, in the hippocampus, fluoxetine reduces fast-spiking inhibitory interneuron activity, which reduces gamma oscillations, independently of its action on monoamines ${ }^{73}$. In the cortex, chronic fluoxetine administration induces a reduction in layer II-III inhibitory interneuron activity which facilitates experience-driven structural dendritic remodelling ${ }^{74}$. A separate study in human primary motor cortex slices demonstrated that fluoxetine-induced reduction of inhibitory tone comes about through suppression of layer II-III monosynaptic excitatory connections from pyramidal cells to inhibitory interneurons, which leaves the monosynaptic output of GABAergic cells unaffected $^{75}$. This layer-specific effect of fluoxetine is interesting in the context of work that demonstrates that early post-stroke 'enriched rehabilitation' is more effective than environmental enrichment or reach training alone as a result of the enhancement of use-dependent plasticity in peri-infarct layer II-III cortex ${ }^{76}$. One idea is that fluoxetine (and other pharmacotherapies) might influence training effects by replicating the biological effects of enriched environments.

\section{Translation: animals to humans and back}

How can this work be translated from animal studies into patients with stroke? Opportunities undoubtedly exist for understanding the biology that underlies regeneration and recovery after stroke further by addressing some of the shortcomings in preclinical models, such as development of biological connectome-style mapping of large-scale axonal, dendritic and synaptic changes, increased use of subcortical white-matter models of stroke and use of older. However, unidirectional translation from preclinical work has not led to dramatic improvements in human stroke recovery. Understanding the biological basis of recovery in humans by navigating the translational pipeline in a bidirectional and iterative ${ }^{79}$ is consequently an urgent priority, because opportunities to augment 
or prolong spontaneous biological recovery would radically alter our understanding of how and when to best promote recovery after stroke. Establishing the nature and duration of a post-stroke critical period in humans is a crucial first step. The questions of whether hyperexcitability or hypoexcitability dominate in the post-stroke period, how long these changes last and whether all patients have the same response all remain to be determined (FIG. 2). Put simply, is the aim to prolong the critical period provided by spontaneous biological recovery or to reinstate it in the chronic phase of stroke, or both? We currently have an explanatory gap between preclinical and human accounts of poststroke recovery mechanisms, which is a barrier to translational work in the recovery. Clinical trials of plasticity-modifying interventions in patients after stroke are currently being implemented without biological targets, which makes treatment of the appropriate patients at the best time almost impossible. Rational therapies require mechanistic approaches, without which large-scale phase-III randomized-control trials of plasticity-modifying interventions are unlikely to succeed ${ }^{80}$.

Animal studies of structural plasticity enhancement suggest that successful outcomes come about through new local and large-scale connectivity. In patients after a stroke, diffusion tensor imaging can be used to examine large white matter tracts ${ }^{81,82}$ but cannot be used to examine axonal terminal fields where a number of important post-stroke changes take place. However, new anatomical connections should bring with them changes in post-stroke functional brain architecture. Functional brain imaging can detect differences in task-related activation patterns that alter in relation to time since stroke ${ }^{83,84}$ and degree of impairment ${ }^{85-87}$. In addition, connectivity patterns after stroke can be assessed either at rest ${ }^{88}$ or during an activity ${ }^{89}$ and these patterns might reflect the combination of new local and large-scale connectivity that is seen in animal models ${ }^{90}$. As yet however, human neuroimaging has not been used to convincingly demonstrate the efficacy of therapies that aim to promote structural plasticity.

Alterations in cortical excitation and inhibition can influence outcome after stroke in animal models and consequently represent exciting and novel therapeutic targets. Studies in humans using transcranial magnetic stimulation ${ }^{91}$, magnetic resonance spectroscopy ${ }^{92}$ and PET ${ }^{93}$ support the idea that GABAergic mechanisms are involved in stroke recovery without resolving the questions posed by work in pre-clinical models, including, as mentioned previously, the time scale of changes in 
cortical excitability, whether hyperexcitability or hypoexcitability predominates (or whether they occur sequentially), and whether all patients have same response. Without answering these questions, designing an effective clinical trial to test any therapeutic intervention that claims to interact with these biological processes is difficult. For example, knowing when an a5-subunit-containing extrasynaptic $\mathrm{GABA}_{\mathrm{A}}$-receptor agonist, fluoxetine, or even noninvasive brain stimulation should be used and who are the patients most liable to respond requires an appropriate biomarker [G] with which to reconcile animal and human accounts of post-stroke recovery ${ }^{94}$. To be truly useful, a biomarker will link observed behaviour to unseen biological phenomena in order to make meaningful mechanistic inferences about that behaviour ${ }^{95}$. In the example of patients with severe upper limb impairment very early after stroke, we have discussed how the observed behaviour (initial impairment) dissociates from the subsequent recovery pathway. Here, we would hope to be able to identify underlying biological phenomena that predict recovery, in a way that observed behaviour cannot, to ask whether failure of recovery is due to failure of the mechanisms underlying spontaneous biological recovery.

A number of tools have been used in humans in an attempt to identify the appropriate biomarker, but most have considerable limitations. For example, transcranial magnetic stimulation is dependent on the presence of evoked potentials in affected muscles, and blood-oxygen-level dependent functional MRI relies on intact neurovascular coupling, limitations that effectively rule out the use of these tools in a large proportion of the patients that we need to study. Magnetic resonance spectroscopy can detect GABA, but it is likely that the majority of the signal is from intracellular, rather than synaptic or extrasynaptic, GABA. PET can assess GABA $_{A}$ ergic activity ${ }^{96,97}$ using flumazenil but this likely reflects cerebral hypoperfusion and neuronal density and integrity ${ }^{98}$, rather than cortical excitability per se. Consequently, interest in the use of neuronal oscillations [G] as biomarkers of the potential for activity-dependent plasticity after stroke is growing ${ }^{94,99,100}$. Neuronal oscillations can be measured noninvasively with magnetoencephalography (MEG) or electroencephalography, which detect the magnetic or electrical fields generated by neuronal activity of the brain ${ }^{101}$. Specifically, MEG measures the summation of postsynaptic fields from pyramidal cells ${ }^{102}$ with excitatory glutamatergic projections, which are reciprocally connected to interneurons 
with inhibitory GABAergic projections. MEG signals are, therefore, dependent on the interaction between inhibition and excitation within cortical microcircuits ${ }^{103}$. For example, resting beta band (15$30 \mathrm{~Hz}$ ) power is enhanced by GABAergic signalling ${ }^{103,104}$. Furthermore, typical movement-related beta desynchronisation is enhanced only by tonic inhibition ${ }^{105,106}$. These neuronal oscillations show high intraindividual reliability ${ }^{107}$ and could serve as appropriate longitudinal biomarkers of net inhibitory and excitatory mechanisms in human cortex after stroke and enable the differentiation between the contribution of phasic and tonic inhibition to the measured signal, thereby providing a window into the mechanisms of activity-dependent plasticity that are important for recovery.

The utility of neuronal oscillations as biomarkers of plasticity mechanisms after stroke is further supported by a number of findings. Firstly, in patients after stroke, poor outcomes are associated with a persistent increase in low-frequency oscillations ${ }^{108}$, similar to those caused by benzodiazepines (a GABA $A_{A}$-agonist that causes phasic inhibition) and tiagabine (a GABA reuptake inhibitor that induces in tonic inhibition) ${ }^{104-106}$, which suggests that inhibitory mechanisms predominate in the perilesional cortex, and impair recovery. Secondly, low beta-rebound in response to tactile finger stimulation (which indicates increased early post-stroke sensorimotor excitability) ${ }^{109}$ and increased sensory map size ${ }^{110}$ predict good recovery in patients with stroke, as in animal models ${ }^{21}$. Lastly, in a single patient with stroke, zolpidem reversed the increases in perilesional theta $(4-10 \mathrm{~Hz})$ and beta oscillations and led to clinical improvement ${ }^{66}$. Zolpidem is pharmacologically interesting in that it has effects on both phasic and tonic GABAergic signalling that can change with dose. The key aspect in this result is that, however zolpidem was acting, the change in neuronal oscillations matched the clinical improvement, which highlights the potential of neuronal oscillations as biomarkers of cortical excitatory-inhibitory balance.

A fundamental understanding of post-stroke recovery has been argued to require the development of computational models of the salient neural processes, including plasticity and learning systems of the brain ${ }^{111}$. This would allow models of underlying biological phenomena to be linked to appropriate behavioural processes. A particular advantage of MEG for this computational neurorehabilitation [G] approach is that the high temporal resolution of the spectral data lends itself to the use of biophysical models. Consequently, mechanistic inferences about post-stroke changes in oscillations 
can be made at both intracortical (mesoscopic) and network (macroscopic) levels (FIG. 3). The model features are neurobiologically motivated ${ }^{112,113}$ so results offer a mechanistically meaningful interpretation at different scales of brain architecture. At the macroscopic level, stroke disrupts functional connections in the peri-infarct region and remotely connected regions, and so investigation of brain-wide network dynamics is important during post-stroke recovery ${ }^{114}$. Modelling of MEG data enables inferences at the cortical network level ${ }^{115}$ and the assessment of both inhibitory and (separately) excitatory effective coupling between cortical motor regions at the same frequency (that is, linear coupling; for example, beta to beta) and different frequencies (that is, non-linear coupling; for example beta to gamma). This assessment is useful as nonlinear coupling is important for functional integration across the brain and could reflect altered structural connectivity across networks that support recovery. Interestingly, inferences can also be made at the cortical microcircuit [G] level ${ }^{112}$. This novel mathematical modelling approach has been validated using local field potentials in animal models where independent pharmacological and microdialysis assays corroborated the modelling results ${ }^{113}$. For example, a novel biophysical model of human primary motor cortex ${ }^{116}$ has been developed to reproduce key neurophysiological characteristics of mouse primary motor cortex ${ }^{117}$. Here, model parameters represent either the strength of connections between pyramidal cells and inhibitory interneurons, or the overall excitability in each population of cells ${ }^{118}$. Ultimately the combination of both scales within a single generative model framework will be possible, to construct a comprehensive model of post-stroke functional architecture. These models can also be applied to local field potential data ${ }^{113}$, providing a way to directly compare, and so validate, recovery mechanisms in future studies in animal models and humans to develop a mechanistic understanding of recovery in humans.

\section{Rehabilitation}

The rationale for understanding how to optimize the post-stroke brain environment is to maximize the effect of behavioural training - which can take the form of physical, cognitive or speech therapy. The presence of a critical period of plasticity advocates for the delivery of high dose and high intensity behavioural training during this window of opportunity to maximize recovery of function by minimising 
impairment ${ }^{18}$. For the upper limb, trials of intensive training that commence before the first 3 months after stroke still provide only modest amounts of therapy and the effect sizes range from minimal to modest ${ }^{119-121}$. One small study started 2-4 weeks after stroke did find that an extra 90 hours of upper limb training ( 3 hours per day for 6 weeks) increased the upper-limb Fugl-Meyer score (a reasonable assessment of motor impairment) by a clinically meaningful extra 12 points compared with those receiving an extra 30 hours $^{122}$. Trials in patients with chronic stroke (in whom over 6 months had elapsed since stroke) have generally delivered up to 30 hours of additional therapy, usually at an hour per day, but have not had dramatic effects on impairment ${ }^{123-125}$. However, one study delivered 300 hours of various upper-limb therapies over 12 weeks to chronic stroke and achieved comparatively large reductions in impairment of 11 points on the Fugl-Meyer scale ${ }^{126}$. Similar changes have been reported in a single-centre service delivering 90 hours of high-dose upper-limb therapy over 3 weeks ${ }^{127}$. In aphasia, the number of hours of therapy also clearly has an effect, with positive studies delivering a mean of 98.4 hours treatment, and negative studies a mean of 43.6 hours $^{128}$. Whether equivalent doses of therapy have an increased effect on impairment if delivered in the early compared with the late post-stroke phase is not yet clear.

Much has been written about what form of behavioural training should be used, how it should be scheduled and what method of delivery is optimal ${ }^{6}$. However, as illustrated by the proportional recovery rule, these deliberations are not currently effecting outcomes at the level of impairment at least, not in the motor domain ${ }^{129}$. The currently used dose and intensity of rehabilitation is probably too low ${ }^{130,131}$, and an increase in both dose and intensity using an appropriate training approach after stroke could lead to the large effect sizes that patients and clinicians want to see. Parallels can be drawn with data from animal studies that demonstrate a threshold of reaching activity, below which little effect on post-stroke outcomes is observed ${ }^{132}$. The amount of therapy (particularly the amount of time on task) has been shown to have a positive influence on outcomes ${ }^{133}$, but these findings are not currently influencing clinical practice.

A key question is whether the lack of a dramatic effect is due to biological factors - in which case, have we already reached the limit of achievable improvements? Alternatively, are we simply not providing enough treatment (at least, not of the correct type or at the right time) or not using the most 
advantageous combinations of treatment? The use of aspirational approaches to investigate what is possible rather than what is pragmatic is vitally important. Current studies tend only to investigate interventions that could be delivered in current health care systems. Only knowledge of the true limits of recovery after stroke, in both the early and chronic phase, will enable the design an appropriate clinical service to achieve maximal recovery in an efficient and cost-effective way. Currently, the resources to deliver intensive early rehabilitation are scarce, and are virtually nonexistent for patients with chronic stroke. In the 1990s, the same was true of acute stroke services, but clinical trials of thrombolysis demonstrated improvements in outcome for stroke patients so compelling ${ }^{134}$ that the way acute stroke care was delivered had to be radically altered to accommodate this new knowledge. In effect, stroke recovery programs need a 'thrombolysis moment', which will only come about through aspirational rather than pragmatic approaches.

\section{Future predictions}

The ability to accurately predict long-term clinical outcomes in patients after stroke is important for a number of reasons. Firstly, outcome prediction is useful to plan treatments and to set goals in a rehabilitation program. Secondly, these predictions will enable clinical trials of restorative treatments to stratify patients in control and treatment groups based on expected outcome, without extremely large numbers of subjects will be required ${ }^{135}$. Thirdly, predictions of long-term outcomes in response to current treatment approaches could become the new benchmark with which to judge novel treatment approaches. In other words, the goal of any new intervention might be to deliver an outcome better than currently predicted, either at an individual or group level.

Currently, the best predictor of long-term outcome - certainly in the motor domain - is initial severity. The limitations of initial severity as an outcome predictor are reflected in the proportional recovery rule, which fails in about half of patients with stroke who present with initially severe impairment ${ }^{14}$. Resolution of the reasons behind the failure to recover in these patients (compared with other patients who have equally severe initial impairment) will not only improve predictive models of long-term outcome, but will reveal the factors important for the recovery process itself. As discussed in previous sections, measures to investigate the mechanisms of post-stroke plasticity in 
patients after a stroke might be usefully incorporated into a predictive model for long-term outcome. Small scale approaches have shown how functional imaging data can readily be incorporated into these models ${ }^{136,137}$.

Any attempt to predict long-term outcome must take into account damage to key brain regions. For example, optimal recovery of movement after stroke requires preservation of anatomical structures that convey sensory signals to the brain, and those that convey motor commands out of the brain, to enable behavioural interventions to drive remapping of sensorimotor functions in surviving brain areas and networks ${ }^{21}$. Indeed, in humans, more extensive corticospinal tract (CST) damage causes greater upper limb impairment ${ }^{138}$; although CST damage correlates with initial upper limb impairment, it can account for some proportion of upper limb outcome over and above that predicted by initial severity ${ }^{15,139}$. Most of this work has been carried out in patients with subcortical strokes and so the effect of damage to widespread cortical areas, especially those required for cognitive functions important for learning such as memory and sustained attention, has not been assessed. Quantification of damage within CST was shown to be poor at accounting for impairment in patients with infarcts involving both subcortical and cortical areas (FIG 4A\&B) ${ }^{140}$. In fact, a combination of cortical motor areas and CST is the most accurate way to account for upper limb motor impairment in a wide range of patients with stroke who have infarcts that involve subcortical and/or cortical regions (FIG 4C\&D) ${ }^{141}$. In the language domain, the Predicting Language Outcome and Recovery After Stroke (PLORAS) system ${ }^{142}$ demonstrates that using similar machine-learning approaches, the individual trajectory of language recovery can be predicted from structural brain scans.

Whether adding information about residual functional architecture will provide independently useful predictive information remains to be seen. In the motor domain, most findings point to lower resting connectivity between primary motor cortices in patients with more motor impairment ${ }^{143}$ and greater corticospinal tract damage ${ }^{144}$. During movement of the affected hand the influence of contralesional to ipsilesional M1 is more inhibitory, but once again, only in more impaired patients ${ }^{89}$. In one study that examined a number of demographic, genetic and brain imaging characteristics of chronic stroke patients undergoing 3 weeks of upper limb robotic training, lower CST damage, absence of cortical 
damage and greater connectivity between primary motor cortices were factors indicating higher chance of clinical improvement ${ }^{145}$.

The incorporation of information about brain structure and function together with readily available clinical information should provide the optimal approach to develop new models that predict longterm outcome after stroke. The size of databases containing this information now needs to increase to maximize the precision with which predictions can be made, because predictive accuracy is liable to be important in determining patient and clinician uptake in utilising this information.

\section{Conclusions}

Great advances have been made in understanding the biological basis of restoration of neurological function after stroke. However, translation into human studies has been slow. Two key elements promote optimal restoration of function after stroke: effective behavioural training that targets impairment as well as function, and treatments that can augment and/or prolong plasticity in the post-stroke critical period of plasticity. Current implementation of new treatments to promote recovery (such as drugs and noninvasive brain stimulation) in phase III trials lacks a clear mechanistic rationale and is, therefore, premature ${ }^{80}$. To achieve progress, mechanistic studies to understand post-stroke mechanisms of plasticity must move into humans with stroke and future investigation in the translational pipeline must become bidirectional and iterative ${ }^{79,95}$. Effective behavioural therapies and appropriate biomarkers of post-stroke plasticity mechanisms are both desperately needed to help understand who and when to treat, and the methodologies to achieve these aims are now readily available. This information must lead to a step-change in how restorative treatments for stroke are delivered. Clinical trial design must take account of the biological mechanisms underlying stroke and should stratify different patient subpopulations, rather than using a 'one size fits all' approach. Attempts to treat impairment in chronic stroke have been disappointing and have not produced the dramatic effect sizes required to transform the field ${ }^{79}$. Targeting the mechanisms that underlie early spontaneous biological recovery in humans represents the mostpromising path to dramatically improve patients' outcomes ${ }^{18}$ and should be prioritized. However, the 
limits of what is possible in chronic stroke have not yet been explored, especially if the delivery of high doses of behavioural therapy in reopened critical periods of plasticity becomes possible.

The author declares no competing interests. 


\section{References}

1. Feigin, V. L. et al. Global and regional burden of stroke during 1990-2010: findings from the Global Burden of Disease Study 2010. Lancet. 383, 245-254 (2014).

2. Lackland, D. T. et al. Factors influencing the decline in stroke mortality: a statement from the American Heart Association/American Stroke Association. Stroke 45, 315-353 (2014).

3. Crichton, S. L., Bray, B. D., McKevitt, C., Rudd, A. G. \& Wolfe, C. D. A. Patient outcomes up to 15 years after stroke: survival, disability, quality of life, cognition and mental health. J. Neurol. Neurosurg. Psychiatry 87, 1091-1098 (2016).

4. Wade, D. T. \& Hewer, R. L. Functional abilities after stroke: measurement, natural history and prognosis. J. Neurol. Neurosurg. Psychiatry 50, 177-182 (1987).

5. Luengo-Fernandez, R., Leal, J. \& Gray, A. UK research spend in 2008 and 2012: comparing stroke, cancer, coronary heart disease and dementia. BMJ Open 5, e006648 (2015).

6. Krakauer, J. W., Carmichael, S. T., Corbett, D. \& Wittenberg, G. F. Getting neurorehabilitation right: what can be learned from animal models? Neurorehabil. Neural Repair 26, 923-931 (2012).

7. Langhorne, P., Coupar, F. \& Pollock, A. Motor recovery after stroke: a systematic review. Lancet Neurol. 8, 741-754 (2009).

8. Lai, S.-M., Studenski, S., Duncan, P. W. \& Perera, S. Persisting consequences of stroke measured by the Stroke Impact Scale. Stroke 33, 1840-1844 (2002).

9. Kwakkel, G., Kollen, B. J., van der Grond, J. \& Prevo, A. J. H. Probability of regaining dexterity in the flaccid upper limb: impact of severity of paresis and time since onset in acute stroke. Stroke 34, 2181-2186 (2003).

10. Broeks, J. G., Lankhorst, G. J., Rumping, K. \& Prevo, A. J. The long-term outcome of arm function after stroke: results of a follow-up study. Disabil. Rehabil. 21, 357-364 (1999).

11. Coupar, F., Pollock, A., Rowe, P., Weir, C. \& Langhorne, P. Predictors of upper limb recovery after stroke: a systematic review and meta-analysis. Clin. Rehabil. 26, 291-313 (2012).

12. Prabhakaran, S. et al. Inter-individual variability in the capacity for motor recovery after ischemic stroke. Neurorehabil. Neural Repair 22, 64-71 (2008). 
13. Zarahn, E. et al. Prediction of motor recovery using initial impairment and fMRI $48 \mathrm{~h}$ poststroke. Cereb. Cortex 21, 2712-2721 (2011).

14. Winters, C., van Wegen, E. E. H., Daffertshofer, A. \& Kwakkel, G. Generalizability of the Proportional Recovery Model for the Upper Extremity After an Ischemic Stroke. Neurorehabil. Neural Repair 29, 614-22 (2014).

15. Byblow, W. D., Stinear, C. M., Barber, P. A., Petoe, M. A. \& Ackerley, S. J. Proportional recovery after stroke depends on corticomotor integrity. Ann. Neurol. 78, 848-59 (2015).

16. Lazar, R. M. et al. Improvement in aphasia scores after stroke is well predicted by initial severity. Stroke 41, 1485-1488 (2010).

17. Nijboer, T. C. W., Kollen, B. J. \& Kwakkel, G. Time course of visuospatial neglect early after stroke: a longitudinal cohort study. Cortex 49, 2021-2027 (2013).

18. Zeiler, S. R. \& Krakauer, J. W. The interaction between training and plasticity in the poststroke brain. Curr. Opin. Neurol. 26, 609-616 (2013).

19. Biernaskie, J., Chernenko, G. \& Corbett, D. Efficacy of rehabilitative experience declines with time after focal ischemic brain injury. J. Neurosci. 24, 1245-1254 (2004).

20. Zeiler, S. R. et al. Paradoxical Motor Recovery From a First Stroke After Induction of a Second Stroke: Reopening a Postischemic Sensitive Period. Neurorehabil. Neural Repair 30, 794-800 (2015).

21. Murphy, T. H. \& Corbett, D. Plasticity during stroke recovery: from synapse to behaviour. Nat. Rev. Neurosci. 10, 861-872 (2009).

22. Carmichael, S. T. Emergent properties of neural repair: elemental biology to therapeutic concepts. Ann. Neurol. 79, 895-906 (2016).

23. Cramer, S. C. \& Chopp, M. Recovery recapitulates ontogeny. Trends Neurosci. 23, 265-271 (2000).

24. Wahl, A.-S. \& Schwab, M. E. Finding an optimal rehabilitation paradigm after stroke: enhancing fiber growth and training of the brain at the right moment. Front. Hum. Neurosci. 8, 381 (2014).

25. Wieloch, T. \& Nikolich, K. Mechanisms of neural plasticity following brain injury. Curr. Opin. Neurobiol. 16, 258-264 (2006). 
26. Carmichael, S. T., Kathirvelu, B., Schweppe, C. A. \& Nie, E. H. Molecular, cellular and functional events in axonal sprouting after stroke. Exp. Neurol. 287, 384-394 (2016).

27. Jin, K. et al. Evidence for stroke-induced neurogenesis in the human brain. Proc. Natl. Acad. Sci. U. S. A. 103, 13198-13202 (2006).

28. Sanin, V., Heeß, C., Kretzschmar, H. A. \& Schüller, U. Recruitment of neural precursor cells from circumventricular organs of patients with cerebral ischaemia. Neuropathol. Appl. Neurobiol. 39, 510-518 (2013).

29. Li, S. et al. An age-related sprouting transcriptome provides molecular control of axonal sprouting after stroke. Nat. Neurosci. 13, 1496-1504 (2010).

30. Li, S. \& Carmichael, S. T. Growth-associated gene and protein expression in the region of axonal sprouting in the aged brain after stroke. Neurobiol. Dis. 23, 362-373 (2006).

31. Benowitz, L. I. \& Carmichael, S. T. Promoting axonal rewiring to improve outcome after stroke. Neurobiol. Dis. 37, 259 (2010).

32. Wahl, A. S. et al. Neuronal repair. Asynchronous therapy restores motor control by rewiring of the rat corticospinal tract after stroke. Science 344, 1250-1255 (2014).

33. Allred, R. P., Maldonado, M. A., Hsu And, J. E. \& Jones, T. A. Training the 'less-affected' forelimb after unilateral cortical infarcts interferes with functional recovery of the impaired forelimb in rats. Restor. Neurol. Neurosci. 23, 297-302 (2005).

34. Kim, S. Y. et al. Experience with the 'good' limb induces aberrant synaptic plasticity in the perilesion cortex after stroke. J. Neurosci. 35, 8604-8610 (2015).

35. Nih, L. R., Carmichael, S. T. \& Segura, T. Hydrogels for brain repair after stroke: an emerging treatment option. Curr. Opin. Biotechnol. 40, 155-163 (2016).

36. Memanishvili, T. et al. Generation of cortical neurons from human induced-pluripotent stem cells by biodegradable polymeric microspheres loaded with priming factors. Biomed. Mater. 11, 025011 (2016).

37. Pendharkar, A. V. et al. Optogenetic modulation in stroke recovery. Neurosurg. Focus 40, E6 (2016).

38. Carmichael, S. T. Brain excitability in stroke: the yin and yang of stroke progression. Arch. Neurol. 69, 161-167 (2012). 
39. Lai, T. W., Zhang, S. \& Wang, Y. T. Excitotoxicity and stroke: identifying novel targets for neuroprotection. Prog. Neurobiol. 115, 157-188 (2014).

40. Clarkson, A. N., Huang, B. S., Macisaac, S. E., Mody, I. \& Carmichael, S. T. Reducing excessive GABA-mediated tonic inhibition promotes functional recovery after stroke. Nature 468, 305-309 (2010).

41. Bavelier, D., Levi, D. M., Li, R. W., Dan, Y. \& Hensch, T. K. Removing brakes on adult brain plasticity: from molecular to behavioral interventions. J. Neurosci. 30, 14964-14971 (2010).

42. Chen, J. L. et al. Structural basis for the role of inhibition in facilitating adult brain plasticity. Nat. Neurosci. 14, 587-594 (2011).

43. Felling, R. J. \& Song, H. Epigenetic mechanisms of neuroplasticity and the implications for stroke recovery. Exp. Neurol. 268, 37-45 (2015).

44. Alia, C. et al. Reducing GABAA-mediated inhibition improves forelimb motor function after focal cortical stroke in mice. Sci. Rep. 6, 37823 (2016).

45. Winship, I. R. \& Murphy, T. H. In vivo calcium imaging reveals functional rewiring of single somatosensory neurons after stroke. J. Neurosci. 28, 6592-6606 (2008).

46. Hagemann, G., Redecker, C., Neumann-Haefelin, T., Freund, H. J. \& Witte, O. W. Increased long-term potentiation in the surround of experimentally induced focal cortical infarction. Ann. Neurol. 44, 255-258 (1998).

47. Takatsuru, Y. et al. Neuronal circuit remodeling in the contralateral cortical hemisphere during functional recovery from cerebral infarction. J. Neurosci. 29, 10081-10086 (2009).

48. Que, M. et al. Changes in $\operatorname{GABA}(\mathrm{A})$ and $\mathrm{GABA}(\mathrm{B})$ receptor binding following cortical photothrombosis: a quantitative receptor autoradiographic study. Neuroscience 93, 1233-1240 (1999).

49. Clarkson, A. N. et al. AMPA receptor-induced local brain-derived neurotrophic factor signaling mediates motor recovery after stroke. J. Neurosci. 31, 3766-3775 (2011).

50. Schäbitz, W.-R. et al. Intravenous brain-derived neurotrophic factor enhances poststroke sensorimotor recovery and stimulates neurogenesis. Stroke 38, 2165-2172 (2007). 
51. Neumann-Haefelin, T., Hagemann, G. \& Witte, O. W. Cellular correlates of neuronal hyperexcitability in the vicinity of photochemically induced cortical infarcts in rats in vitro. Neurosci. Lett. 193, 101-104 (1995).

52. Schiene, K. et al. Neuronal hyperexcitability and reduction of GABAA-receptor expression in the surround of cerebral photothrombosis. J. Cereb. Blood Flow Metab. 16, 906-914 (1996).

53. Zeiler, S. R. et al. Medial premotor cortex shows a reduction in inhibitory markers and mediates recovery in a mouse model of focal stroke. Stroke 44, 483-489 (2013).

54. Lake, E. M. R. et al. The effects of delayed reduction of tonic inhibition on ischemic lesion and sensorimotor function. J. Cereb. Blood Flow Metab. (2015). doi:10.1038/jcbfm.2015.86

55. Clarkson, A. N. Perisynaptic GABA Receptors The Overzealous Protector. Adv. Pharmacol. Sci. 2012, 708428 (2012).

56. Sakuma, M., Hyakawa, N., Kato, H. \& Araki, T. Time dependent changes of striatal interneurons after focal cerebral ischemia in rats. J. Neural Transm. 115, 413-422 (2008).

57. Kharlamov, E. A., Downey, K. L., Jukkola, P. I., Grayson, D. R. \& Kelly, K. M. Expression of GABA A receptor alpha1 subunit mRNA and protein in rat neocortex following photothrombotic infarction. Brain Res. 1210, 29-38 (2008).

58. Hsu, W.-Y., Cheng, C.-H., Liao, K.-K., Lee, I.-H. \& Lin, Y.-Y. Effects of repetitive transcranial magnetic stimulation on motor functions in patients with stroke: a meta-analysis. Stroke. 43, 1849-1857 (2012).

59. Kang, N., Summers, J. J. \& Cauraugh, J. H. Transcranial direct current stimulation facilitates motor learning post-stroke: a systematic review and meta-analysis. J. Neurol. Neurosurg. Psychiatry (2015). doi:10.1136/jnnp-2015-311242

60. Fritsch, B. et al. Direct current stimulation promotes BDNF-dependent synaptic plasticity: potential implications for motor learning. Neuron 66, 198-204 (2010).

61. Bonaiuto, J. J. \& Bestmann, S. Understanding the nonlinear physiological and behavioral effects of tDCS through computational neurostimulation. Prog. Brain Res. 222, 75-103 (2015).

62. de Berker, A. O., Bikson, M. \& Bestmann, S. Predicting the behavioral impact of transcranial direct current stimulation: issues and limitations. Front. Hum. Neurosci. 7, 613 (2013). 
63. Prokic, E. J. et al. Cortical oscillatory dynamics and benzodiazepine-site modulation of tonic inhibition in fast spiking interneurons. Neuropharmacology 95, 192-205 (2015).

64. Hiu, T. et al. Enhanced phasic GABA inhibition during the repair phase of stroke: a novel therapeutic target. Brain awv360 (2015). doi:10.1093/brain/awv360

65. Cohen, L., Chaaban, B. \& Habert, M.-O. Transient improvement of aphasia with zolpidem. N. Engl. J. Med. 350, 949-950 (2004).

66. Hall, S. D. et al. GABA(A) alpha-1 subunit mediated desynchronization of elevated low frequency oscillations alleviates specific dysfunction in stroke--a case report. Clin. Neurophysiol. 121, 549-555 (2010).

67. Phillips, J. P., Devier, D. J. \& Feeney, D. M. Rehabilitation pharmacology: bridging laboratory work to clinical application. J. Head Trauma Rehabil. 18, 342-356 (2003).

68. Chollet, F. et al. Fluoxetine for motor recovery after acute ischaemic stroke (FLAME): a randomised placebo-controlled trial. Lancet Neurol. 10, 123-130 (2011).

69. Mead, G. E. et al. Selective serotonin reuptake inhibitors (SSRIs) for stroke recovery. Cochrane Database Syst. Rev. 11, CD009286 (2012).

70. Maya Vetencourt, J. F. et al. The antidepressant fluoxetine restores plasticity in the adult visual cortex. Science 320, 385-388 (2008).

71. Ng, K. L. et al. Fluoxetine Maintains a State of Heightened Responsiveness to Motor Training Early After Stroke in a Mouse Model. Stroke 46, 2951-2960 (2015).

72. Puig, M. V., Watakabe, A., Ushimaru, M., Yamamori, T. \& Kawaguchi, Y. Serotonin modulates fast-spiking interneuron and synchronous activity in the rat prefrontal cortex through 5-HT1A and 5-HT2A receptors. J. Neurosci. 30, 2211-2222 (2010).

73. Méndez, P., Pazienti, A., Szabó, G. \& Bacci, A. Direct alteration of a specific inhibitory circuit of the hippocampus by antidepressants. J. Neurosci. 32, 16616-16628 (2012).

74. Chen, J. L. et al. Structural basis for the role of inhibition in facilitating adult brain plasticity. Nat. Neurosci. 14, 587-594 (2011).

75. Komlósi, G. et al. Fluoxetine (prozac) and serotonin act on excitatory synaptic transmission to suppress single layer 2/3 pyramidal neuron-triggered cell assemblies in the human prefrontal cortex. J. Neurosci. 32, 16369-16378 (2012). 
76. Clarke, J., Langdon, K. D. \& Corbett, D. Early poststroke experience differentially alters periinfarct layer II and III cortex. J. Cereb. Blood Flow Metab. 34, 630-637 (2014).

77. Luria, A. Restoration of function after brain injury. (Pergammon Press, 1963).

78. Luria, A., Naydin, V., Tsvetkova, L. \& Vinarskaya, E. in Handbook of Clinical Neurology 3, 368433 (North Holland Publishing Company, 1963).

79. Cumberland Consensus Working Group et al. The future of restorative neurosciences in stroke: driving the translational research pipeline from basic science to rehabilitation of people after stroke. Neurorehabil. Neural Repair 23, 97-107 (2009).

80. Ward, N. S. Getting lost in translation. Curr. Opin. Neurol. 21, 625-627 (2008).

81. Schulz, R. et al. Assessing the integrity of corticospinal pathways from primary and secondary cortical motor areas after stroke. Stroke. 43, 2248-2251 (2012).

82. Schulz, R. et al. White matter integrity of premotor-motor connections is associated with motor output in chronic stroke patients. Neurolmage Clin. 7, 82-86 (2015).

83. Ward, N. S., Brown, M. M., Thompson, A. J. \& Frackowiak, R. S. J. Longitudinal changes in cerebral response to proprioceptive input in individual patients after stroke: an FMRI study. Neurorehabil. Neural Repair 20, 398-405 (2006).

84. Ward, N. S., Brown, M. M., Thompson, A. J. \& Frackowiak, R. S. J. Neural correlates of motor recovery after stroke: a longitudinal fMRI study. Brain 126, 2476-2496 (2003).

85. Ward, N. S., Brown, M. M., Thompson, A. J. \& Frackowiak, R. S. J. Neural correlates of outcome after stroke: a cross-sectional fMRI study. Brain 126, 1430-1448 (2003).

86. Ward, N. S. et al. Motor system activation after subcortical stroke depends on corticospinal system integrity. Brain 129, 809-819 (2006).

87. Ward, N. S., Brown, M. M., Thompson, A. J. \& Frackowiak, R. S. J. The influence of time after stroke on brain activations during a motor task. Ann. Neurol. 55, 829-834 (2004).

88. Wang, L. et al. Dynamic functional reorganization of the motor execution network after stroke. Brain 133, 1224-1238 (2010).

89. Grefkes, C. et al. Cortical connectivity after subcortical stroke assessed with functional magnetic resonance imaging. Ann. Neurol. 63, 236-246 (2008). 
90. Dijkhuizen, R. M., Zaharchuk, G. \& Otte, W. M. Assessment and modulation of resting-state neural networks after stroke. Curr. Opin. Neurol. 27, 637-643 (2014).

91. Swayne, O. B. C., Rothwell, J. C., Ward, N. S. \& Greenwood, R. J. Stages of motor output reorganization after hemispheric stroke suggested by longitudinal studies of cortical physiology. Cereb. Cortex 18, 1909-1922 (2008).

92. Blicher, J. U. et al. GABA Levels Are Decreased After Stroke and GABA Changes During Rehabilitation Correlate With Motor Improvement. Neurorehabil. Neural Repair 29, 278-286 (2015).

93. Kim, Y. K., Yang, E. J., Cho, K., Lim, J. Y. \& Paik, N.-J. Functional Recovery After Ischemic Stroke Is Associated With Reduced GABAergic Inhibition in the Cerebral Cortex: A GABA PET Study. Neurorehabil. Neural Repair 28, 576-583 (2014).

94. Ward, N. S. Using oscillations to understand recovery after stroke. Brain 138, 2811-2813 (2015).

95. Bernhardt, J. et al. Moving rehabilitation research forward: Developing consensus statements for rehabilitation and recovery research. Int. J. Stroke 11, 454-458 (2016).

96. Heiss, W.-D. et al. Permanent Cortical Damage Detected by Flumazenil Positron Emission Tomography in Acute. Stroke 29, 454-461 (1998).

97. Kim, Y. K., Yang, E. J., Cho, K., Lim, J. Y. \& Paik, N.-J. Functional Recovery After Ischemic Stroke Is Associated With Reduced GABAergic Inhibition in the Cerebral Cortex: A GABA PET Study. Neurorehabil. Neural Repair (2014). doi:10.1177/1545968313520411

98. Baron, J.-C., Yamauchi, H., Fujioka, M. \& Endres, M. Selective neuronal loss in ischemic stroke and cerebrovascular disease. J. Cereb. Blood Flow Metab. 34, 2-18 (2014).

99. Rabiller, G., He, J.-W., Nishijima, Y., Wong, A. \& Liu, J. Perturbation of Brain Oscillations after Ischemic Stroke: A Potential Biomarker for Post-Stroke Function and Therapy. Int. J. Mol. Sci. 16, 25605-25640 (2015).

100. Paggiaro, A. et al. Magnetoencephalography in Stroke Recovery and Rehabilitation. Front. Neurol. 7, 35 (2016).

101. Proudfoot, M., Woolrich, M. W., Nobre, A. C. \& Turner, M. R. Magnetoencephalography. Pract. Neurol. 14, 336-343 (2014). 
102. Murakami, S. \& Okada, Y. Contributions of principal neocortical neurons to magnetoencephalography and electroencephalography signals. J. Physiol. 575, 925-936 (2006).

103. Yamawaki, N., Stanford, I. M., Hall, S. D. \& Woodhall, G. L. Pharmacologically induced and stimulus evoked rhythmic neuronal oscillatory activity in the primary motor cortex in vitro. Neuroscience 151, 386-395 (2008).

104. Nutt, D. et al. Differences between magnetoencephalographic (MEG) spectral profiles of drugs acting on GABA at synaptic and extrasynaptic sites: a study in healthy volunteers. Neuropharmacology 88, 155-163 (2015).

105. Hall, S. D. et al. The role of GABAergic modulation in motor function related neuronal network activity. Neurolmage 56, 1506-1510 (2011).

106. Muthukumaraswamy, S. D. et al. The effects of elevated endogenous GABA levels on movement-related network oscillations. Neurolmage 66, 36-41 (2013).

107. Espenhahn, S., de Berker, A. O., van Wijk, B. C. M., Rossiter, H. E. \& Ward, N. S. Movementrelated beta oscillations show high intra-individual reliability. Neurolmage (2016). doi:10.1016/j.neuroimage.2016.12.025

108. Laaksonen, K. et al. Alterations in spontaneous brain oscillations during stroke recovery. PloS One 8, e61146 (2013).

109. Laaksonen, K. et al. Effect of afferent input on motor cortex excitability during stroke recovery. Clin. Neurophysiol. 123, 2429-2436 (2012).

110. Roiha, K. et al. Reorganization of the primary somatosensory cortex during stroke recovery. Clin. Neurophysiol. 122, 339-345 (2011).

111. Reinkensmeyer, D. J. et al. Computational neurorehabilitation: modeling plasticity and learning to predict recovery. J. Neuroengineering Rehabil. 13, 42 (2016).

112. Moran, R. J. et al. Bayesian estimation of synaptic physiology from the spectral responses of neural masses. Neurolmage 42, 272-284 (2008).

113. Moran, R. J. et al. Dynamic causal models and physiological inference: a validation study using isoflurane anaesthesia in rodents. PloS One 6, e22790 (2011). 
114. Ward, N. S. Does neuroimaging help to deliver better recovery of movement after stroke? Curr. Opin. Neurol. 28, 323-329 (2015).

115. Chen, C.-C. et al. Nonlinear coupling in the human motor system. J. Neurosci. 30, 8393-8399 (2010).

116. Bhatt, M. B. et al. Computational modelling of movement-related beta-oscillatory dynamics in human motor cortex. Neurolmage 133, 224-232 (2016).

117. Weiler, N., Wood, L., Yu, J., Solla, S. A. \& Shepherd, G. M. G. Top-down laminar organization of the excitatory network in motor cortex. Nat. Neurosci. 11, 360-366 (2008).

118. Muthukumaraswamy, S. D. et al. Broadband cortical desynchronization underlies the human psychedelic state. J. Neurosci. 33, 15171-15183 (2013).

119. Winstein, C. J. et al. Effect of a Task-Oriented Rehabilitation Program on Upper Extremity Recovery Following Motor Stroke: The ICARE Randomized Clinical Trial. JAMA 315, 571-581 (2016).

120. Kwakkel, G. et al. Effects of Unilateral Upper Limb Training in Two Distinct Prognostic Groups Early After Stroke: The EXPLICIT-Stroke Randomized Clinical Trial. Neurorehabil. Neural Repair (2016). doi:10.1177/1545968315624784

121. Harris, J. E., Eng, J. J., Miller, W. C. \& Dawson, A. S. A self-administered Graded Repetitive Arm Supplementary Program (GRASP) improves arm function during inpatient stroke rehabilitation: a multi-site randomized controlled trial. Stroke 40, 2123-2128 (2009).

122. Han, C., Wang, Q., Meng, P. \& Qi, M. Effects of intensity of arm training on hemiplegic upper extremity motor recovery in stroke patients: a randomized controlled trial. Clin. Rehabil. 27, 75-81 (2013).

123. Lo, A. C. et al. Robot-assisted therapy for long-term upper-limb impairment after stroke. $N$. Engl. J. Med. 362, 1772-1783 (2010).

124. Lang, C. E. et al. Dose-response of task-specific upper limb training in people at least 6 months post stroke: A Phase II, single-blind, randomized, controlled trial. Ann. Neurol. (2016). doi:10.1002/ana.24734

125. Klamroth-Marganska, V. et al. Three-dimensional, task-specific robot therapy of the arm after stroke: a multicentre, parallel-group randomised trial. Lancet Neurol. 13, 159-166 (2014). 
126. McCabe, J., Monkiewicz, M., Holcomb, J., Pundik, S. \& Daly, J. J. Comparison of robotics, functional electrical stimulation, and motor learning methods for treatment of persistent upper extremity dysfunction after stroke: a randomized controlled trial. Arch. Phys. Med. Rehabil. 96, $981-990$ (2015).

127. Ward NS, Suhaimei U, Strawson A, O’Neill C, Briggs J, Brander F, Kelly K. The Queen Square intensive upper limb rehabilitation programme. Int. J. Stroke 11(4S): S14 (2016).

128. Bhogal, S. K., Teasell, R. \& Speechley, M. Intensity of aphasia therapy, impact on recovery. Stroke34, 987-993 (2003).

129. Krakauer, J. W. \& Marshall, R. S. The proportional recovery rule for stroke revisited. Ann. Neurol. (2015). doi:10.1002/ana.24537

130. Lang, C. E. et al. Observation of amounts of movement practice provided during stroke rehabilitation. Arch. Phys. Med. Rehabil. 90, 1692-1698 (2009).

131. Bernhardt, J., Dewey, H., Thrift, A. \& Donnan, G. Inactive and alone: physical activity within the first 14 days of acute stroke unit care. Stroke 35, 1005-1009 (2004).

132. MacLellan, C. L. et al. A critical threshold of rehabilitation involving brain-derived neurotrophic factor is required for poststroke recovery. Neurorehabil. Neural Repair 25, 740-748 (2011).

133. Lohse, K. R., Lang, C. E. \& Boyd, L. A. Is more better? Using metadata to explore doseresponse relationships in stroke rehabilitation. Stroke 45, 2053-2058 (2014).

134. Tissue plasminogen activator for acute ischemic stroke. The National Institute of Neurological Disorders and Stroke rt-PA Stroke Study Group. N. Engl. J. Med. 333, 1581-1587 (1995).

135. Winters, C., Heymans, M. W., van Wegen, E. E. H. \& Kwakkel, G. How to design clinical rehabilitation trials for the upper paretic limb early post stroke? Trials 17, 468 (2016).

136. Saur, D. et al. Early functional magnetic resonance imaging activations predict language outcome after stroke. Brain 133, 1252-1264 (2010).

137. Rehme, A. K. et al. Identifying Neuroimaging Markers of Motor Disability in Acute Stroke by Machine Learning Techniques. Cereb. Cortex N. Y. N 1991 (2014). doi:10.1093/cercor/bhu100

138. Stinear, C. M. \& Ward, N. S. How useful is imaging in predicting outcomes in stroke rehabilitation? Int. J. Stroke. 8, 33-37 (2013). 
139. Bigourdan, A. et al. Early Fiber Number Ratio Is a Surrogate of Corticospinal Tract Integrity and Predicts Motor Recovery After Stroke. Stroke 47, 1053-1059 (2016).

140. Park, C.-H., Kou, N. \& Ward, N. S. The contribution of lesion location to upper limb deficit after stroke. J. Neurol. Neurosurg. Psychiatry (2016). doi:10.1136/jnnp-2015-312738

141. Rondina, J. M., Filippone, M., Girolami, M. \& Ward, N. S. Decoding post-stroke motor function from structural brain imaging. Neurolmage Clin. 12, 372-380 (2016).

142. Seghier, M. L. et al. The PLORAS Database: A data repository for Predicting Language Outcome and Recovery After Stroke. Neurolmage (2015). doi:10.1016/j.neuroimage.2015.03.083

143. Carter, A. R. et al. Upstream dysfunction of somatomotor functional connectivity after corticospinal damage in stroke. Neurorehabil. Neural Repair 26, 7-19 (2012).

144. Carter, A. R. et al. Resting interhemispheric functional magnetic resonance imaging connectivity predicts performance after stroke. Ann. Neurol. 67, 365-375 (2010).

145. Burke Quinlan, E. et al. Neural function, injury, and stroke subtype predict treatment gains after stroke. Ann. Neurol. 77, 132-145 (2015).

146. Lindau, N. T. et al. Rewiring of the corticospinal tract in the adult rat after unilateral stroke and anti-Nogo-A therapy. Brain. 137, 739-756 (2014).

147. Meininger, V. et al. Safety, pharmacokinetic, and functional effects of the nogo-a monoclonal antibody in amyotrophic lateral sclerosis: a randomized, first-in-human clinical trial. PloS One 9, e97803 (2014).

148. Cramer, S. C. et al. Safety, pharmacokinetics, and pharmacodynamics of escalating repeat doses of GSK249320 in patients with stroke. Stroke 44, 1337-1342 (2013).

149. Pizzorusso, T. et al. Reactivation of ocular dominance plasticity in the adult visual cortex. Science 298, 1248-1251 (2002).

150. Gherardini, L., Gennaro, M. \& Pizzorusso, T. Perilesional treatment with chondroitinase ABC and motor training promote functional recovery after stroke in rats. Cereb. Cortex 25, 202-212 (2015).

151. Anderson, M. A. et al. Astrocyte scar formation aids central nervous system axon regeneration. Nature 532, 195-200 (2016). 
152. Overman, J. J. et al. A role for ephrin-A5 in axonal sprouting, recovery, and activitydependent plasticity after stroke. Proc. Natl. Acad. Sci. U. S. A. 109, E2230-2239 (2012).

153. Zai, L. et al. Inosine alters gene expression and axonal projections in neurons contralateral to a cortical infarct and improves skilled use of the impaired limb. J. Neurosci. 29, 8187-8197 (2009).

154. Dachir, S. et al. Inosine improves functional recovery after experimental traumatic brain injury. Brain Res. 1555, 78-88 (2014).

155. Zai, L. et al. Inosine augments the effects of a Nogo receptor blocker and of environmental enrichment to restore skilled forelimb use after stroke. J. Neurosci. 31, 5977-5988 (2011).

156. Li, S. et al. GDF10 is a signal for axonal sprouting and functional recovery after stroke. Nat. Neurosci. 18, 1737-1745 (2015).

157. Kalladka, D. \& Muir, K. W. Where are we in clinical applications of stem cells in ischaemic stroke? Adv. Clin. Neurosci. Rehabil. 16, (2016).

158. Azad, T. D., Veeravagu, A. \& Steinberg, G. K. Neurorestoration after stroke. Neurosurg. Focus 40, E2 (2016).

159. Tornero, D. et al. Human induced pluripotent stem cell-derived cortical neurons integrate in stroke-injured cortex and improve functional recovery. Brain 136, 3561-3577 (2013).

160. Steinberg, G. K. et al. Clinical Outcomes of Transplanted Modified Bone Marrow-Derived Mesenchymal Stem Cells in Stroke: A Phase 1/2a Study. Stroke 47, 1817-1824 (2016).

161. Kalladka, D. et al. Human neural stem cells in patients with chronic ischaemic stroke (PISCES): a phase 1, first-in-man study. Lancet 388, 787-796 (2016). 


\section{Figure legends}

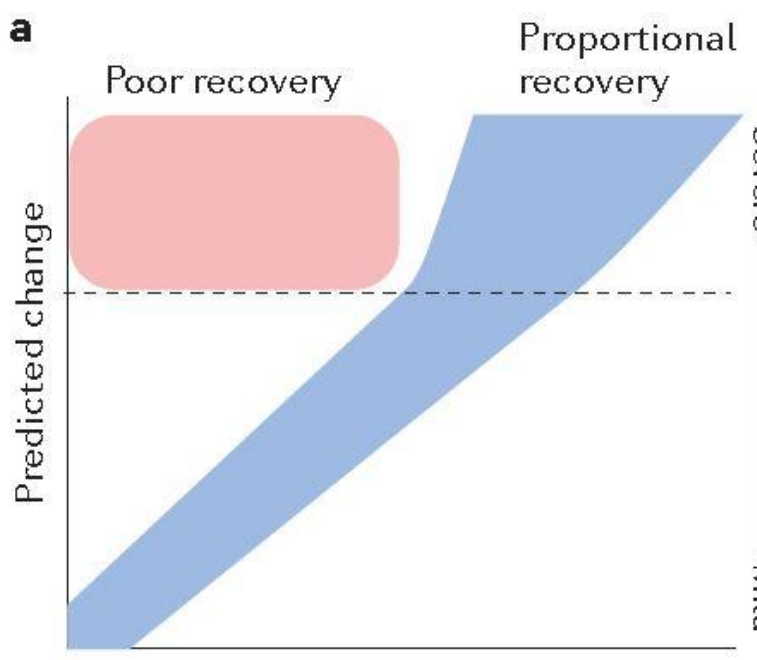

Observed change b

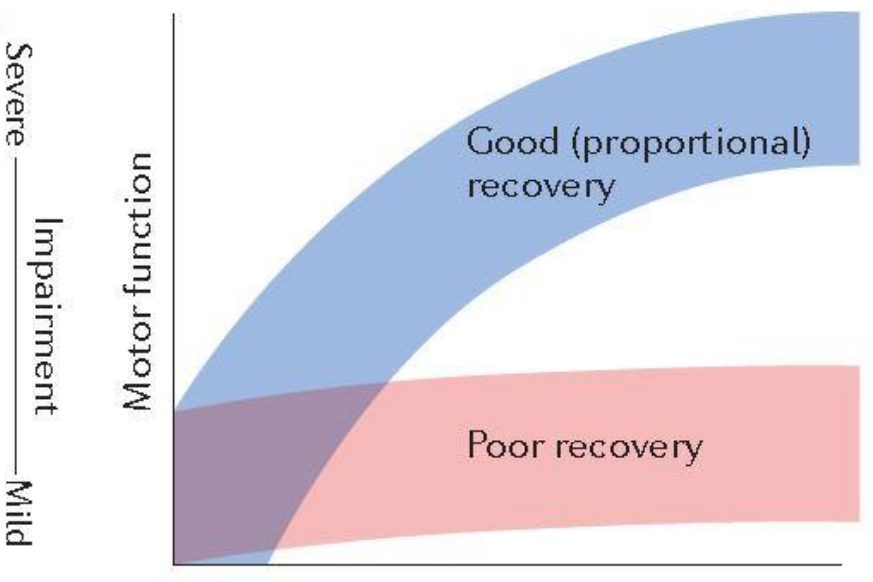

Time poststroke

Figure 1 | Proportional motor recovery in the upper limb. a | Predicted change in upper-limb impairment plotted against observed change at 3 months post-stroke. Patients are predicted to regain approximately $70 \%$ of their initial deficit by 3 months after stroke. Patients in the blue area have proportional recovery as predicted. Patients in the red area recover less well than predicted. Patients in dotted black box all have initially severe levels of impairment, with roughly equal numbers of good (proportional) and poor recoverers. b | Illustration of different recovery curves of patients with initially severe upper limb impairment - who have recovery as predicted (blue) or poor recovery (red). This suggests that the factors important for recovery are different from those responsible for initial severity. 


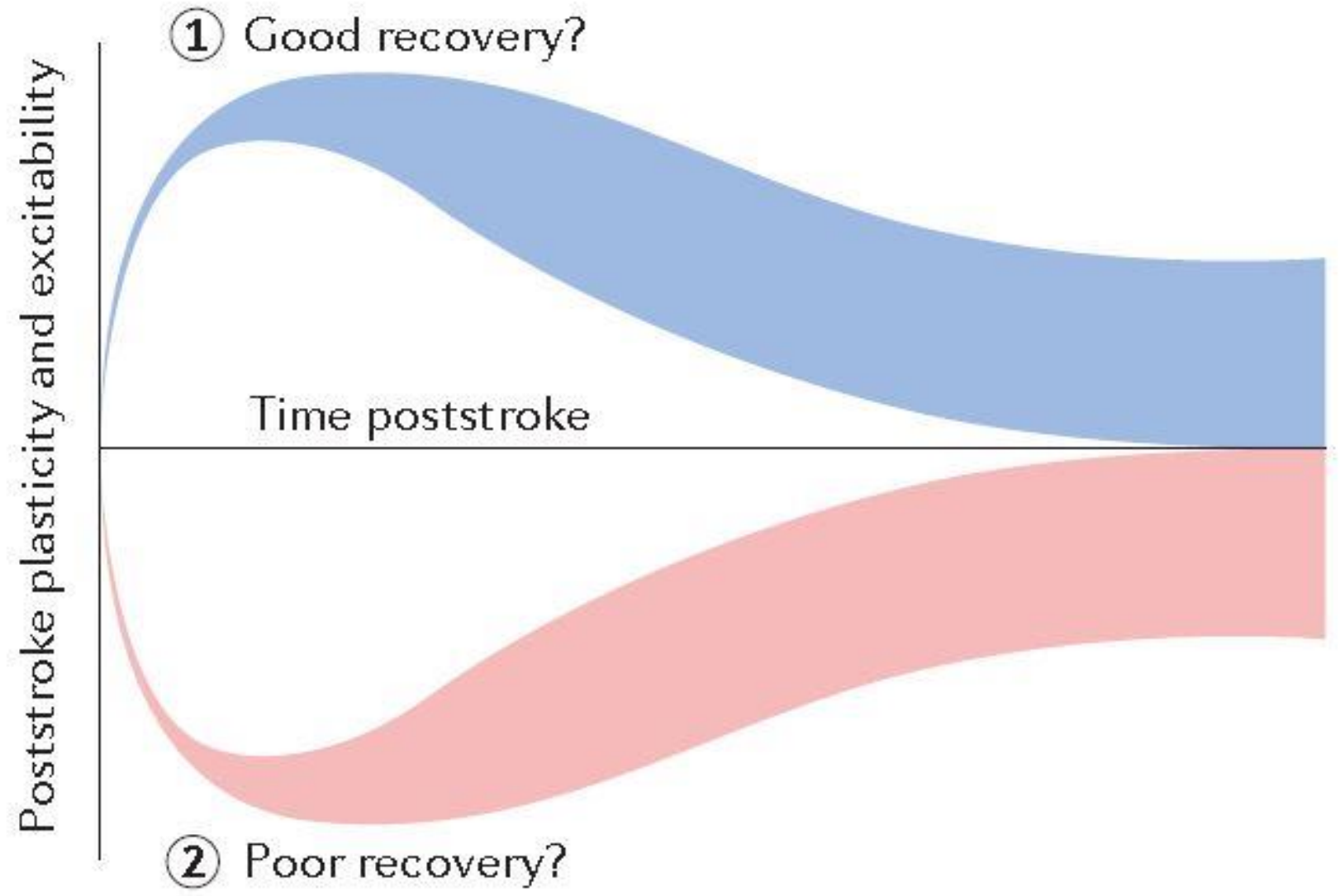

Figure 2 / Post-stroke plasticity and recovery. Evidence from animal models of stroke points to both (1) an upregulation of neuronal growth-promoting factors and an increase in neuronal excitability (blue area), both of which would create an environment that would encourage recovery of function, and (2) an upregulation of neuronal growth-inhibiting factors and an decrease in neuronal excitability (red area) both of which would create an environment that would limit recovery of function. Whether these processes occur after stroke in humans is unknown. Further work is required to determine which of these processes predominates, how long each one lasts, and whether the same processes occur in all patients to the same degree. This knowledge is crucial for determining the optimal timing for the introduction of both behavioural and plasticity-enhancing treatments. 


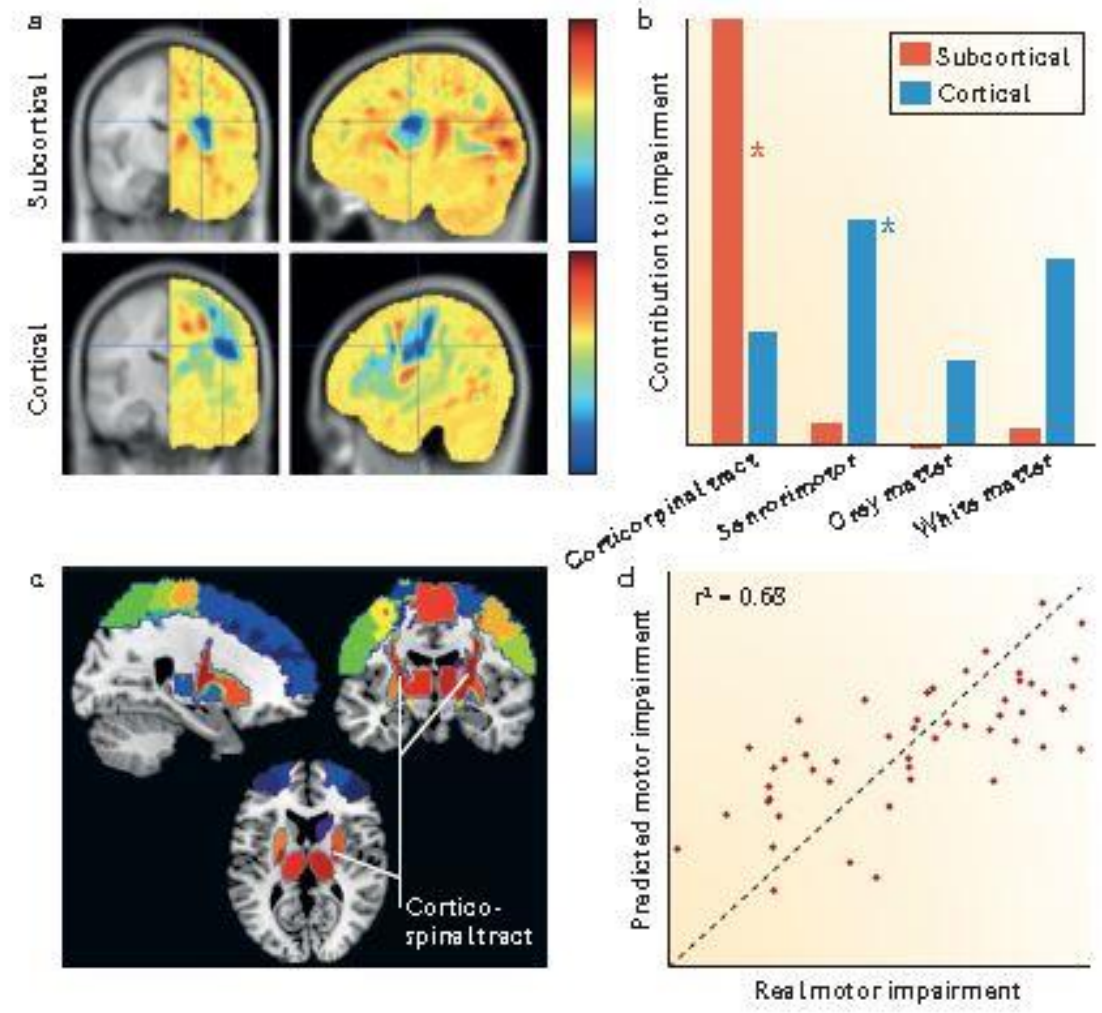

Figure 3 / Characterization of anatomical damage. a | Brain maps derived from patients with chronic stroke caused by damage to subcortical brain regions ('subcortical') with or without extension to the cortex ('cortical'). The colour scale represents the voxel-wise 'impairment weighting', i.e. the contribution that damage in a brain area is likely to make to motor impairment. Blue represents the areas where damage is highly likely to cause motor impairment: these areas extend into cortical regions for the 'cortical' patients. Analysis was performed using a multivoxel pattern analysis tool PRoNTo. $b$ | The mean impairment weightings for four different regions of interest in patients with cortical or subcortical stroke damage. Whereas damage within corticospinal tract accounted for impairment in the subcortical group (red asterisk), damage in sensorimotor areas accounted for more impairment in the cortical group (blue asterisk)136. Damage in a whole brain mask of white matter and grey matter contributed to impairment in patients with cortical but not subcortical stroke damage. c | A brain map of cortical and subcortical brain regions important for sensorimotor function (colours represent different anatomical regions from the automated anatomical labelling atlas), together with a corticospinal tract map78. d | A machine-learning regression approach showed that damage to 
voxels contained in the map in part c accounted for $68 \%$ of motor impairment in 50 patients with chronic stroke, as illustrated in the graph of predicted motor impairment plotted against real motor impairment in these patients. The same analysis using only the corticospinal tract region of interest accounted for only $42 \%$ of the motor impairment, suggesting that knowledge of damage to a range of motor related brain structures, not just corticospinal tract, is important for predicting outcome 137. Parts $a$ and $b$ modified with permission from BMJ Publishing group Itd. (C) Park, C.-H., Kou, N. \& Ward, N. S. J. Neurol. Neurosurg. Psychiatry (2016). Parts c and d modified with permission from Elsevier (c) Rondina, J. et al. Neurolmage Clin. 12, 372-380 (2016). 


\section{Glossary}

Proportional recovery rule: The amount of function regained after stroke is a proportion of the initial deficit. For example, by 3 months patients will regain $~ 70 \%$ of the upper limb motor function that had been lost on day 3 post-stroke.

Spontaneous biological recovery: Recovery occurring in the first few weeks and months after stroke, attributable to enhanced post-stroke plasticity mechanisms. Recovery is rapid, occurs at the level of impairment and generalizes beyond the tasks that are used in post-stroke training, compared with improvements seen in the chronic phase of stroke.

Biomarkers: Indicators of disease state that can be used clinically as a measure reflecting underlying molecular or cellular processes that might be difficult to measure directly in humans, and can be used to predict recovery or treatment response ${ }^{95}$.

Neuronal oscillations: Rhythmic fluctuations in activity generated either spontaneously or in response to stimuli by neural tissue in the CNS. Entrained oscillations in multiple neurons and neural networks are thought to form a critical interface between cellular activity and large-scale functions in the CNS.

Computational neurorehabilitation: a newly emerging field aimed at mathematical modelling of plasticity and learning to understand and improve recovery of individuals with neurologic impairment.

Cortical microcircuit: The pattern of connections between specific excitatory and inhibitory neurons in the cortex.

Hemispatial neglect: Reduced awareness of stimuli on one side of space, even though sensory loss might be absent. 


\section{Box 1 | Factors that block inhibition of neuronal regeneration}

\section{Myelin-associated proteins}

Myelin-associated proteins such as Reticulon4 (also known as NogoA), myelin-associated glycoprotein (MAG), and myelin-associated oligodendrocyte basic protein have been shown to block neuronal regeneration. An anti-NogoA antibody has been used in preclinical models both of stroke and of spinal cord injury, and leads to improved recovery profiles. Sprouting is often seen across the midline, either at the level of brain stem or spinal cord. Lindau and colleagues ${ }^{146}$ found that rats treated with anti-NogoA antibody recovered motor control after sensorimotor cortex ablation because intact corticospinal tract had extensively sprouted across the midline into the denervated spinal hemicord, which led to a somatotopic anatomical and functional side switch in the projection of adult corticospinal neurons. The safety of anti-NogoA antibodies has been tested in patients with spinal cord injury ${ }^{24}$ and amyotrophic lateral sclerosis ${ }^{147}$ and anti-MAG has been tested in patients with stroke ${ }^{148}$.

\section{Extracellular matrix proteins}

Chondroitin sulphate proteoglycans mediate the inhibitory properties of perineuronal nets and are known to block axon growth. Cortical infarcts lead to reduced density of PNNs in peri-infarct cortex, maximal at 30 days post-lesion ${ }^{44}$. The enzyme chondroitinase $A B C$ can reinstate critical period plasticity via the inactivation of chondroitin sulphate proteoglycans and therefore $\mathrm{PNNs}^{149}$. In a rat model of stroke, chondroitinase $A B C$ helped restore motor function after both acute and delayed administration ${ }^{150}$. However, extracellular matrix proteins are not always inhibitory, for example, the prevention of astrocytic scar formation can reduce stimulated axon regrowth. e.g. preventing astrocytic scar formation can reduce stimulated axon regrowth ${ }^{151}$.

\section{Growth cone inhibitors}

Neuronal regeneration can also be inhibited by molecules that inhibit the axonal growth cone, such as semaphorins and ephrins. Ephrin-A5 is induced in astrocytes in peri-infarct cortex, which leads to inhibition of axonal sprouting. When ephrin-A5 signalling is blocked, then motor training is more liable to promote recovery ${ }^{152}$. In this case, sprouting leads to a new pattern of reparative axonal projections in motor-related cortices of the ipsilesional hemisphere. 


\section{Box 2 | Factors that promote neuronal regeneration}

\section{Inosine}

The naturally occurring purine nucleoside inosine has been reported to enhance axon growth and improve outcomes in a preclinical model of stroke. Inosine promotes axonal collateral sprouting into areas that have lost their normal innervation, such as the corticospinal tract after stroke ${ }^{153}$ or hippocampus after experimental traumatic brain injury ${ }^{154}$. Furthermore, inosine can augment the effects of anti-NogoA antibody (BOX 1) to restore skilled forelimb use after stroke ${ }^{155}$.

\section{Growth and differentiating factor 10 (GDF10)}

The gene encoding GDF10 is highly upregulated in the axonal regenerative transcriptome induced in peri-infarct neurons and promotes functionally useful axonal sprouting ${ }^{156}$.

\section{Stem cells}

Increasingly, interest has been shown in the use of stem cell therapy to promote recovery after stroke ${ }^{157}$. The two main lines of stem cell therapies are endogenous (promoting the production of existing neural stem cells) or exogenous (transplanted from another source) ${ }^{158}$. Over the past few years, research has explored how to reprogram adult human somatic cells to induced pluripotent stem cells thereby producing patient-specific cells for autologous transplantation ${ }^{159}$. Rather than restoring lost tissue, stem cells could act as stimulants for trophic factors and modulators of immunological and inflammatory changes after stroke. Trials of exogenous cells in humans have proved safe and claims have been made for improved clinical outcomes in patients with chronic stroke ${ }^{160,161}$.

\section{Box 3 | Pharmacotherapy for stroke recovery: an historical perspective}

The idea of pharmacotherapy for stroke recovery is not recent. Early work in this field was performed in 1963 by Alexander Luria and colleagues in soldiers with head injuries ${ }^{77}$. In addition to cell death, Luria et al. proposed that symptoms could be induced by functional inhibition of intact neurons and that "removal of the diaschisis, restoration of synaptic conduction or to use another term, 'deblocking'” might be helpful ${ }^{78}$. The investigators proposed that this task could be achieved by the combination of two approaches. First, the administration of a pharmacological agent (generally 
anticholinesterases) "capable of removing inhibition, modifying mediator metabolism and restoring disturbed synaptic conduction"78, and second by methods of training which promote 'de-blocking', the essence of which is "that by means of various methods the level of excitability in certain functional systems is raised and the corresponding functions are "de-inhibited"'78. The general concepts have a familiar ring compared to current concepts, but although the underlying mechanisms might now be more apparent than in the past, the clinical outcomes have not advanced a great deal. 